\title{
Experimental investigation of low-frequency pulsed Lorentz force influence on the motion of Galinstan melt
}

\author{
Diana A. Musaeva ${ }^{\mathrm{a}, *}$, Vladimir K. Ilyin ${ }^{\mathrm{a}}$, Vadims Geža ${ }^{\mathrm{b}}$, Egbert Baake \\ ${ }^{a}$ Kazan State Power Engineering University, 51, Krasnoselskaya St., 420066 Kazan, Russian Federation \\ ${ }^{\mathrm{b}}$ University of Latvia, 8 Zellu St., LV-1002 Riga, Latvia \\ ${ }^{c}$ Leibniz University of Hannover, Welfengarten 1, 30167 Hannover, Germany
}

Available online 20 August 2016

\begin{abstract}
The paper presents the results of the numerical and physical experiments, aimed at assessing the influence of pulsed force of electromagnetic field on the melt motion and the fluid velocities. The experiment was performed on the eutectic alloy Galinstan in the cylindrical volume, where an ultrasonic Doppler velocimeter was employed for velocity measurements under conditions of pulsed and steady EM field application. A numerical simulation of the melt flow, forced by the steady EM force, involved a 2D axisymmetric model. The $k-\varepsilon$ turbulence model was used to obtain the information about the melt velocities. The verification of the numerical model was carried out for the steady case. The effects of pulsed and steadily applied Lorentz force were compared using the physical experiment. An intensity of the velocity pulsations in Galinstan for the pulsed frequencies in the range from 0.05 to $1 \mathrm{~Hz}$ considerably exceeded the values measured in the permanently stirred melt. For higher values of pulsed frequencies (from 1 to $10 \mathrm{~Hz}$ ) the effect decreases and only slightly differs from the influence of the permanently applied Lorentz force.
\end{abstract}

Copyright $\odot$ 2016, St. Petersburg Polytechnic University. Production and hosting by Elsevier B.V.

This is an open access article under the CC BY-NC-ND license. (http://creativecommons.org/licenses/by-nc-nd/4.0/)

Keywords: Pulsed; Modulated Lorentz force; Electromagnetic stirring; Mathematical simulation; Galinstan.

\section{Introduction}

The motion of fluid under transient conditions and the effect of such conditions on the behavior of heat and mass transfer in the flow remain today an important research topic, as in certain cases, transient effects cause the heat transfer intensification, and in this con-

\footnotetext{
* Corresponding author.

E-mail addresses: musaeva.d.a@gmail.com (D.A. Musaeva), ilinwk@rambler.ru (V.K. Ilyin), vadims.geza@gmail.com (V. Geža), baake@etp.uni-hannover.de (E. Baake).
}

text, flow behavior under the changing conditions is of practical interest.

One of the widely used methods for intensifying the heat transfer in fluid by vigorously stirring it is in applying low-frequency pulses to the fluid (generated by mechanical, pneumatic, ultrasonic and other means $[1,2])$. The frequency range of the applied vibrations is determined by the physical parameters of the fluid, the feasibility of applying the impact, the purposes of the application and by other factors.

Material processing using an induction furnace is an energy-consuming process that has gained extensive 
use in metallurgy; efficient stirring of the melt is a key component of this process affecting both the quality of the produced material and its costs.

The type of induction furnace most frequently used is equipped with a cylindrical crucible which is surrounded by a cooled inductor. In general, the inductor is evenly distributed along the height of the crucible. The melt in these devices is exposed to electromagnetic (EM) induction, and the flow is controlled without direct interaction with the fluid, which is a significant advantage (considering the aggressiveness of liquid metals). The alternating current flow in the inductor produces an alternating magnetic field which interacts with the conducting material, causing Joule heat to generate in the bulk of the metal and Lorentz forces governing the melt motion to emerge.

The structure of the flow and the heat and mass transfer in a cylindrical volume are well-studied numerically and experimentally, including works by the authors of this paper, in scaled and full-size furnace models [3-6]. The flow is an intensive turbulent motion forming two toroidal eddies, located one above the other, with opposite directions of rotation. Researchers have observed a significant transfer of heat and mass in the region separating the eddies. Flow intensity depends directly on the values of the current in the inductor and the frequency of the alternating EM field, which are adjustable parameters.

Since electromagnetic melt stirring is widely used in industry, a number of studies $[7,8]$ have been dedicated to effectively controlling liquid metal flow in the crucible of the induction furnace and to intensifying the heat and mass transfer in the fluid under stirring. Another method for intensifying the stirring that is more popular due its higher efficiency is using a discrete power supply, namely, applying electromagnetic pulses [6,9-11]. The parameters of the generated field are chosen based on the data on the structure of fluid flow, which allows to improve the momentum of the melt without increasing the mean flow velocity. Thus, it is possible to stir the melt more intensely without increasing the input power compared with the flow generated under a continuously applied alternating magnetic field.

The pulse effect of the EM field on the melt can be accomplished by interrupting different types of magnetic fields, e.g, traveling, rotating, and constant.

This study considers the effect of the EM field force on melt flow in a scaled model of an induction crucible furnace with an alternator running at a certain pulse frequency $f_{p}=0.05-10 \mathrm{~Hz}$ periodically switched on and off. The melt-stirring intensity is compared with

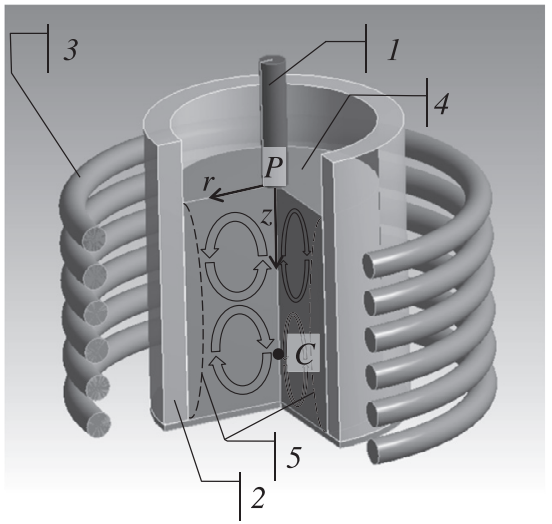

Fig. 1. Schematic of the experimental setup: sensor 1, container 2, inductor 3, melt 4, distribution of the Lorentz force 5; flow lines in the melt are shown; $P$ is the point where the sensor is fixed, $C$ is the peak point on the profile of the measured axial velocity for the lower eddy (see Fig. 2 and the description in the text).

the flow of liquid metal under a constantly applied force of the EM field $\left(f_{p}=0, s t f\right)$.

\section{Experimental setup}

The goal of the paper was to experimentally study the effect of the low-frequency pulsed force of the EM field (the Lorentz force) on the flow of a eutectic alloy called Galinstan, consisting of gallium (68\%), indium (20\%) and tin (12\%). The melting temperature of Galinstan allows measuring the flow parameters at room temperature. The materials used in the experiments and their physical properties are listed in Table 1.

The cylindrical container in which the melt was placed was made of Plexiglas. The container was surrounded by the inductor uniformly distributed over the height of the melt (Fig. 1).

The experimental setup was so designed that the information on the flow parameters under transient conditions could be obtained by an ultrasound Doppler velocity meter.

Fig. 1 schematically shows the characteristic distribution of the Lorentz force (5), induced in the melt in an alternating magnetic field, and the flow lines indicating the main directions in which the fluid moves under the influence of the EM field force. As previously mentioned, the structure of the melt flow in the cylindrical volume at the above-noted density distribution of the Lorentz force is the circulation of two toroidal eddies. The density of the force applied to the melt oscillates at a frequency which is double the frequency of the EM field. At the same time, the change 
Table 1

Values of the basic parameters of the studied objects.

\begin{tabular}{lllll}
\hline Number & Parameter & Notation & Unit & Value \\
\hline $\mathrm{Ga}_{0.68} \mathrm{In}_{0.20} \mathrm{Sn}_{0.12}$ alloy & & & & \\
1 & Density & $\rho$ & $\mathrm{kg} / \mathrm{m}^{3}$ & 6440 \\
2 & Dynamic viscosity & $\mu$ & $\mathrm{Pas}$ & 0.0024 \\
3 & Thermal conductivity & $\lambda$ & $\mathrm{W} /(\mathrm{m} \cdot \mathrm{K})$ & 16.5 \\
4 & Melting temperature & $T$ & $\mathrm{~K}$ & $\sim 275$ \\
5 & Speed of sound in the medium & $V_{c}$ & $\mathrm{~m} / \mathrm{s}$ & 2730 \\
6 & Acoustic impedance of the medium & $Z$ & $\mathrm{~kg} /\left(\mathrm{m}^{2} \mathrm{~s}\right)$ & 18.9 \\
7 & Electric conductivity & $\sigma$ & $\mathrm{S} / \mathrm{M}$ & $3.46 \cdot 10^{6}$ \\
8 & Radius of cylindrical melt volume & $R$ & $\mathrm{~mm}$ & 31 \\
9 & Melt height & $H$ & $\mathrm{~mm}$ & 70 \\
Copper inductor & Electric conductivity & & & \\
10 & Number of turns & $N$ & $\mathrm{~S} / \mathrm{m}$ & $1.74 \cdot 10^{8}$ \\
11 & Turn diameter & - & 6 \\
12 & Distance between turns & $D$ & $\mathrm{~mm}$ & 8 \\
13 & Inductor radius & $n$ & $\mathrm{~mm}$ & 4 \\
14 & & $R_{\text {ind }}$ & $\mathrm{mm}$ & 61 \\
\hline
\end{tabular}

in the Lorentz force density can be neglected, as liquid metals are slow to respond (due to their inertness) to impacts with a frequency higher than $4 \mathrm{~Hz}$ [10]. Varying the frequency of the EM field leads to a change in the depth to which the field can penetrate the conducting liquid (the skin depth) and in the area of the field's action. The density value of the EM field force decreases exponentially within the skin depth whose thickness also depends on the characteristics of the medium acted upon. The thickness of the skin depth can be described by the relation

$\delta=\left(\pi f_{a c} \mu_{m} \sigma\right)^{-1 / 2}$,

where $f_{a c}$ is the frequency with which the direction of the EM field changes, $\mu_{m}=1.256 \cdot 10^{-6} \mathrm{~N} / \mathrm{A}^{2}$ is the magnetic constant, $\sigma$ is the electric conductivity of the medium.

The $\delta$ value for Galinstan was $22 \mathrm{~mm}$; for the inside diameter $D$ of the container equal to $62 \mathrm{~mm}$, this made up a substantial portion of the melt volume which the field affects by setting the fluid in motion.

The flow parameters were determined experimentally using the Doppler velocity meter. As the name implies, its operating principles are based on the Doppler effect, i.e., on changing the frequency of the ultrasonic wave emitted and received by the sensor as the wave is reflected by the microparticles distributed in the fluid. The particle drift velocity within the ultrasonic beam is determined by the difference between the frequencies of the emitted and the reflected waves. This method is widely used for finding the flow parameters in low-melting liquid metals and model fluids $[5,12,13]$.
In the experiment described, a DOP3010 ultrasonic Doppler velocity meter (Signal Processing SA, Switzerland) was used. During the measurements, the sensor ( $8 \mathrm{~mm}$ in diameter) was placed directly into the fluid through the free surface to a depth of 1$2 \mathrm{~mm}$ and fixed in that position throughout the whole experiment. The frequency of the signal transmitted by the sensor was $1428 \mathrm{~Hz}$ at the speed of sound in the melt equal to $2730 \mathrm{~m} / \mathrm{s}$ (see Table 1). The sensor was secured on the cylindrical volume axis (point $P$ $(r=0 \mathrm{~mm})$ in Fig. 1) for measuring the axial component of the velocity. This velocity component was predominant in the flow structure under investigation. At the same time, the velocity had a negative value if the flow parallel to the signal beam was directed toward the sensor and a positive one if the particles in the fluid were moving away from the sensor.

The experiment used two modes of applying the EM field force to the flow. In the first case, we measured the melt flow velocities under a continuous current in the inductor for the $f_{a c}$ values ranging from 50 to $250 \mathrm{~Hz}$. Given that $f_{a c} \gg 4 \mathrm{~Hz}$, the effect on the melt was considered steady (from now on, the EM field parameters are denoted by the index $s t$ ).

In the second case, we considered the motion of fluid under the action of the pulsed Lorentz force (parameters with the index $p$ ). As the force was modulated, the current in the inductor was interrupted to generate pulses with the frequency $f_{p}$. If we denote the period of time during which the EM field force acts on the melt as $T_{a c t}$, and the duration of the pause in which the Lorentz force is absent and the fluid moves under the influence of inertia as $T_{0}$, the expression for 
determining the frequency and the period of the pulses can be written as follows:

$f_{P}=\frac{1}{T_{P}}, T_{P}=T_{a c t}+T_{0}, \psi=\frac{T_{0}}{T_{a c t}}$,

where $\psi$ is the on/off ratio of the pulses of the EM field force.

According to the law of electromagnetic induction, the Lorentz force that emerges in the melt upon application of an alternating magnetic field is expressed as

$\mathbf{F}_{\mathrm{EM}}=\mathbf{j} \times \mathbf{B}$.

Let us denote the value of the resulting density of the EM field force for the steady action as $F_{E M}^{s t}$. Then $F_{E M}^{P}$ is the density of the force acting on the flow during the time $T_{a c t}$. The value of $F_{E M}^{P}$ was set so that, given the $T_{0}$ half-cycle during which the EM field did not act on the melt, the following integral equality between the steady and the pulse modes would be preserved:

$\frac{1}{T_{P}} \int_{0}^{T_{P}} F_{E M}^{P} d T=F_{E M}^{s t}$.

We experimentally studied the effect of transient conditions for the following frequency values of the modulated pulses: $f_{p}=0.05 ; 0.10 ; 0.20 ; 0.50 ; 0.80 ; 1$; $2 ; 5 ; 8 ; 10 \mathrm{~Hz}$. The pulse on/off ratio was $\psi=1\left(T_{a c t}\right.$ $\left.=T_{0}\right)$. We also examined the action of the constant Lorentz force on the flow. For each mode, 2000 instantaneous axial velocity profiles were recorded for 200 seconds, at a resolution of 80 dots; the profiles were then averaged over the whole measurement time. As a result of the measurements, we obtained an axial velocity distribution along the cylinder axis. Velocity fluctuations were also recorded by measuring the standard deviation of the velocity from its mean value.

\section{Mathematical model of melt flow under a constant Lorentz force}

To select the optimal parameters of the EM field, and to obtain a preliminary estimate of the flow intensity, we have developed and experimentally verified a two-dimensional axisymmetric mathematical model of melt flow under a steady action. Simulating the motion of the melt in a magnetic field was a dual task and was done in two stages:

I. Harmonic electromagnetic analysis (via the Mechanical APDL of the Ansys software package);

II. Simulation of fluid motion using the standard $k$ - $\varepsilon$ turbulence model (via the Fluent package).
The first stage of the simulation resulted in obtaining the density distribution of the Lorentz force for the given parameters of the alternating current in the inductor. This force is symmetric with respect to the axis of the cylindrical volume; as noted above. Only the radial component was taken into account in the simulation, since its value is considerably higher than those of the axial and the angular components.

The electromagnetic processes are described by Maxwell's equations, which can be written as follows in differential form in a quasi-stationary setting:

$$
\begin{aligned}
\nabla \cdot \mathbf{E} & =0, \nabla \cdot \mathbf{B}=0, \\
\nabla \times \mathbf{E} & =-\frac{\partial \mathbf{B}}{\partial t}, \nabla \times \mathbf{B}=-\mu_{m} \mathbf{j},
\end{aligned}
$$

where $\mathbf{E}$ is the electric field vector, $\mathbf{B}$ is the magnetic flux density, $\mathbf{j}$ is the current density, $t$ is the time, $\mu_{m}$ is the permeability of the medium.

In the general case, the following system of equations is solved in order to describe the magnetohydrodynamic processes:

$\mathbf{j}=\sigma[\mathbf{E}+\mathbf{v} \times \mathbf{B}]$,

$\frac{\partial \mathbf{B}}{\partial t}=\nabla \times[\mathbf{v} \times \mathbf{B}]+\frac{1}{\mu_{m} \sigma} \nabla^{2} \mathbf{B}$,

$\nabla \mathbf{j}=0$.

Here $\mathbf{v}$ is the local velocity of the conducting medium.

In this system, Eq. (3) describes the diffusion of the magnetic field into an incompressible conducting fluid. Given that the motion of the conducting fluid does not affect the magnetic field, and if we take the electrical conductivity of the medium for a constant, as the Magnetic Reynolds number

$\mathbf{R e}_{m}=L V \mu_{m} \sigma \ll 1$,

( $L$ and $V$ are the characteristic length and velocity), then this expression can be also represented as a diffusion equation

$\frac{\partial \mathbf{B}}{\partial t}=\lambda_{m a g} \nabla^{2} \mathbf{B}$,

$\operatorname{div} \mathbf{B}=0$,

where $\lambda_{\text {mag }}=\left(\mu_{m} \sigma\right)^{-1}$ is the diffusion coefficient.

A conducting object placed in the EM field experiences the action of the force dependent on the magnitude of the magnetic field. The bulk density of the EM field force is expressed by formula (1). Using the relationship between the current and the curl of the 
magnetic field and the formula for the curl of the vector field, we can rewrite the expression in the form

$\mathbf{F}_{E M}=\frac{1}{\mu_{m}}(\mathbf{B} \nabla) \mathbf{B}-\nabla\left(\frac{\mathbf{B}^{2}}{2 \mu_{m}}\right)$.

The second term in this expression is a gradient of a scalar quantity and indicates the potential force that has no effect on the motion of the fluid. The first term describes the body force setting the fluid in motion.

Simulating the hydrodynamic process involved integrating the density distribution of the EM field force as a boundary condition into a mesh created to simulate the motion of fluid using a standard $k-\varepsilon$ turbulence model in the Fluent package designed for calculating hydrodynamic processes. The computational mesh consisted of ten thousand elements; it was refined near the cylindrical surface and in the bottom region so that the normalized coordinate $y+$ of the computational point closest to the wall fell in the $30 \leq$ $y+\leq 60$ range corresponding to the turbulence model used [14]. In order to factor the effect that the presence of a solid wall had on the flow into the calculations, the Enhanced Wall Treatment option was chosen. The relationship between the pressure in the melt and its velocity was calculated using the SIMPLE algorithm. The intense vorticity of the flow was taken into account by interpolating the pressure in the calculation by the PRESTO! scheme. However, the model used did not take into account the changes in the shape of the free surface under the effect of the magnetic field and the flows within the volume of the melt. Because of this, the slip and impermeability conditions were imposed on the upper boundary. Fig. 2 shows the model with the imposed boundary conditions at both stages of the dual simulation.

Thus, the calculated Navier-Stokes equations for an incompressible conducting fluid which include the density of the EM field force as the driving force can be expressed in the following way in differential form:

$\operatorname{div} \mathbf{v}=0$

$\rho \frac{\partial \mathbf{v}}{\partial t}+\rho(\mathbf{v} \nabla) \mathbf{v}=-\nabla \mathbf{p}+\operatorname{div}\left(2 \mu_{e f} S\right)+\mathbf{F}_{E M}$.

Here $\mu_{\text {ef }}$ is the effective viscosity, defined as the sum of the laminar and the turbulent viscosity, i.e., $\mu_{e f}=\mu+\mu_{t} ; S$ is the strain rate tensor.

Simulating the melt flow in the induction furnace with an alternating EM field using the $k-\varepsilon$ turbulence model allows to calculate the average velocities and the flow structure that are in substantial agreement with the experimental data $[3,15,16]$.

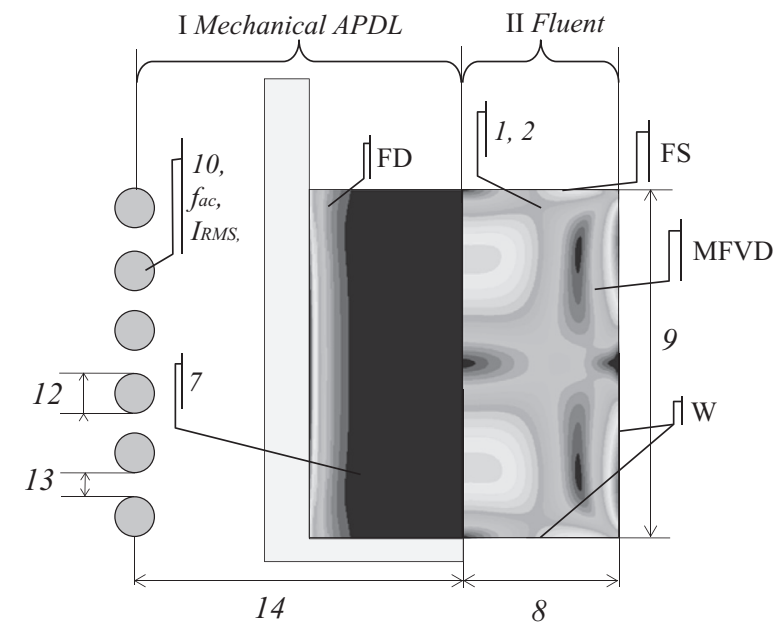

Fig. 2. Two-dimensional model used in the numerical simulation of melt flow under the influence of a constant Lorentz force (FD is its distribution); MFVD is the distribution of the mean flow velocity; I, II are the modeling stages. The figure shows the simulation parameters (the position numbers correspond to the ones listed in Table 1), as well as boundary conditions (aside from FD) set during the simulation: W (wall), FS (free surface).

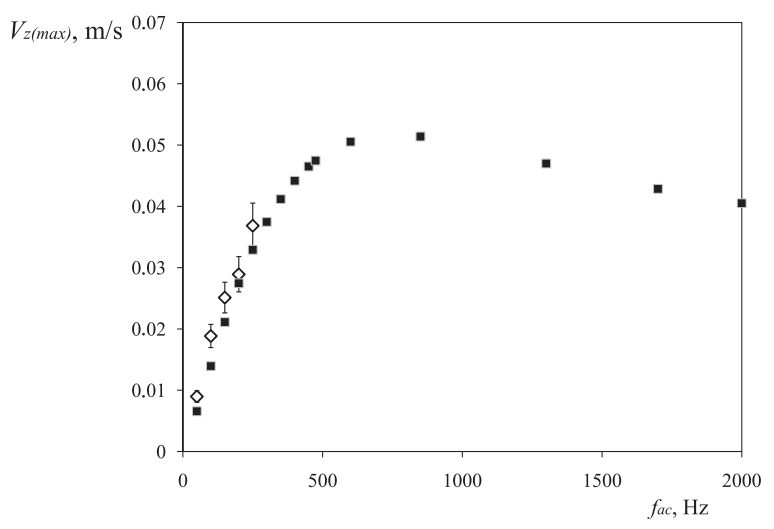

Fig. 3. Maximum axial velocity of the melt on the axis of its cylindrical volume $(r=0)$ versus the frequency of the alternating current in the inductor, obtained numerically (black squares) and experimentally (open diamonds). The Lorentz force is constant, $I_{\mathrm{RMS}}=200 \mathrm{~A}$. The computational model used is $2 \mathrm{D}-k-\varepsilon$. The error range is $\pm 5 \%$.

\section{Verification of the mathematical model}

To check the validity of the model we numerically studied the motion of the molten metal for a fixed current value $I_{\mathrm{RMS}}=200 \mathrm{~A}$ and for a variety of $f_{a c}$ values (Fig. 3). The graph demonstrates that the changes in the maximum axial velocities on the axis of the cylindrical volume depend on the AC frequency in the inductor.

This functional relationship reflects the effectiveness of applying the EM field to the flow and is usually expressed in terms of the dimensionless ratio 


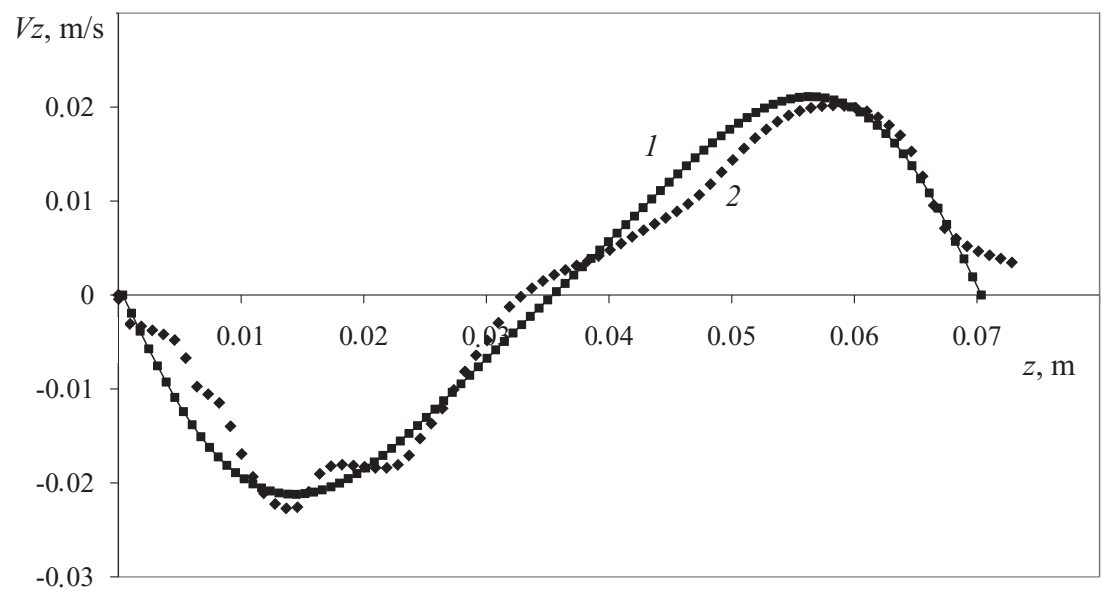

Fig. 4. Distributions of axial velocity $(r=0)$ over the depth of the melt, obtained numerically $(1)$ and experimentally (2). Steady conditions, $f_{a c}=150 \mathrm{~Hz}, I_{\mathrm{RMS}}=200 \mathrm{~A}$.

of the cylinder diameter to the thickness of the skin depth $(D / \delta)$. The shape of the curve shown, containing a peak and then monotonic behavior of the function after it, is typical for the interaction between the alternating magnetic field and the conducting material in it. [17]

The numerical experiment also yielded a characteristic flow pattern and the distribution of the axial velocity on the cylinder axis $(r=0)$ time-averaged over the duration of the experiment exposed to the constant Lorentz force (Figs. 2 and 4). The profile corresponds to the theoretical model of flow formation under the action of an alternating magnetic field, as two maximum values of the measured velocity $V_{z}$, corresponding to the divergent flows of two toroidal eddies along the axis, are present in the graph.

In addition, the curve can be seen passing through the $x$-axis at $35 \mathrm{~mm}$, which corresponds to the region of minimal axial velocities (eddies rotation) in the central region of the melt volume [3-5]. Also notice the agreement between the maximum velocities obtained numerically and experimentally.

For the physical experiment, the following parameters were chosen in accordance with the technical characteristics of the equipment to create the EM field in steady mode: the AC frequency in the inductor $f_{a c}=150 \mathrm{~Hz}$ and the effective current value $I_{\text {RMS }}=200 \mathrm{~A}$.

\section{Melt flow under pulse conditions}

To estimate the effect of the changing frequency $f_{P}$ on the behavior of the fluid flow, we compared the intensities of the flow velocity fluctuations for pulse frequencies in the range from 0.05 to $10 \mathrm{~Hz}$. For this purpose, we calculated the standard deviations of the measured axial velocity from its mean value over the whole duration of the experiment. The results of these calculations allowed to estimate how actively the fluid responded to a pulse or a constant external action (Fig. 5). The solid horizontal line on the graph marks the value of the measured velocity fluctuation for the flow developing under the influence of the constant Lorentz force.

Analysis of the experimental data has shown that a pulsed EM field force with a frequency in the range from 0.50 to $10 \mathrm{~Hz}$ has little effect on the flow: the values of the velocity fluctuations are close to the one obtained for the steady case. This result can be explained by the inertia of the fluid, which is consistent with the experimental data of other studies $[10,15]$; such conditions can be regarded as quasi-steady.

The most significant influence on melt flow was from the pulsed modes of the Lorentz force in the range of $f_{p}=0.05-0.20 \mathrm{~Hz}$. At the same time, the maximum of axial velocity fluctuations is observed for the frequency $f_{p}=0.10 \mathrm{~Hz}$.

It is well known, that in the melt flow driven by an alternating magnetic field, there exist the velocity pulsations with a relatively low period. As shown by the authors of [3], this period depends on the EM force strength and the geometry of the vessel containing the fluid, and as mentioned in Ref. [4], it is associated with the revolution period of toroidal eddy structures in the flow $\left(T_{c h}\right)$, which are generated by an alternating electromagnetic field. The characteristic frequency $f_{c h}$ (the notation adopted by the authors of Ref. [18]) corresponding to this period was determined as fol- 


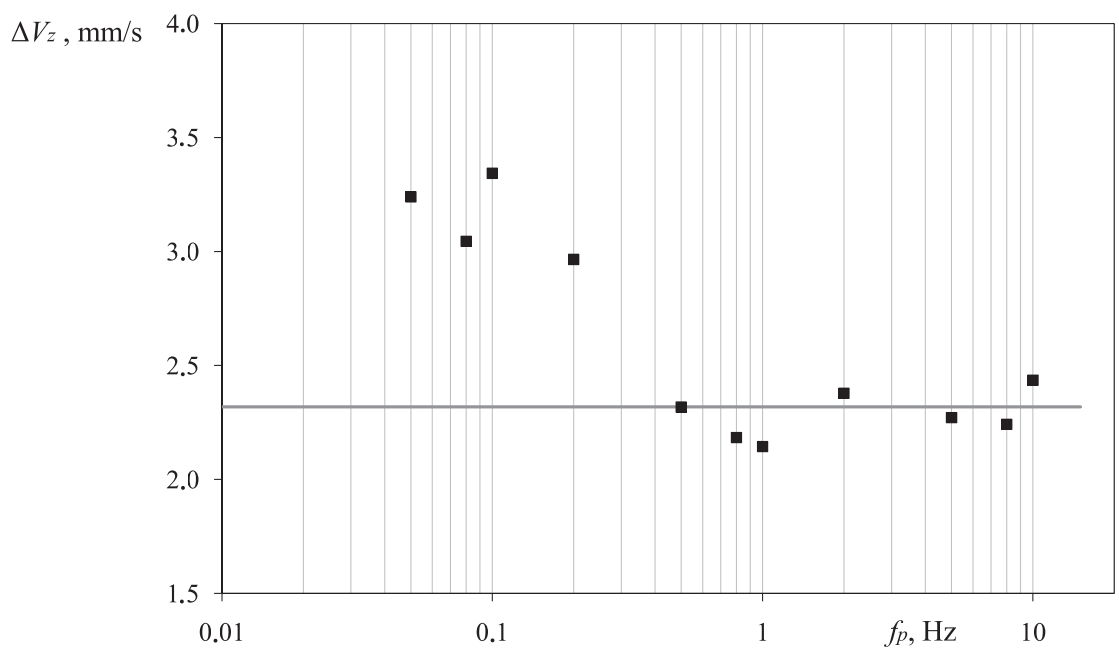

Fig. 5. The effect of the modulation frequency of the Lorentz force on the intensity of the flow velocity fluctuations. We compare the standard deviations of the axial velocity from its mean value $\left(\Delta V_{z}\right)$ under steady-state (horizontal line) and pulsed (points, $\left.\psi=1\right)$ impacts.

lows:

$$
\begin{aligned}
T_{P c h} & =\frac{L_{c h}}{V_{c h}} ; L_{c h}=2 \pi \cdot \frac{r_{r}+r_{z}}{2} ; \\
V_{c h} & =\frac{V_{\max , \text { wall }}+V_{\max , \text { axis }}}{2} ; f_{c h}=\frac{1}{T_{c h}},
\end{aligned}
$$

where $r_{r}$ and $r_{z}$ are the radial and the axial sizes of the eddies, $V_{c h}$ is the characteristic flow velocity.

For the experiment described in this paper, the value of the axial velocity, averaged over the measurement time, for the point $C$ on the container axis with the coordinates $r=0 \mathrm{~mm}, z=60 \mathrm{~mm}$ (see Fig. 1) served as the characteristic velocity. This point approximately corresponds to the maximum on the profile of the measured axial velocity for the lower eddy (see Fig. 2). The velocity in point $C$ under steady action was measured several times in order to reduce measurement errors. The value of the velocity $V_{c h}$ in point $C$, developed under the influence of the constant Lorentz force, amounted to $0.018 \mathrm{~m} / \mathrm{s}$.

Using the above expressions for determining characteristic flow parameters (4) yielded the values for the period and the frequency of eddy revolution (Table 2).

It is assumed that the presence of a maximum in Fig. 5 is because the pulse frequency of the EM field force approaches the characteristic frequency of eddy revolution in the flow. This increases the efficiency of applying the pulses and contributes to an increase in the values of the local flow velocities in the melt. It is reasonable to assume that the real maximum of the velocity fluctuation for the pulsed mode of external action is near the value $f_{p} \sim 0.17 \mathrm{~Hz}$.
Table 2

Computed results of the characteristic parameters for the Galinstan melt flow.

\begin{tabular}{llll}
\hline Parameter & Notation & Unit & Value \\
\hline Eddy size & & & \\
radial & $r_{r}$ & $\mathrm{~cm}$ & 1.55 \\
axial & $r_{z}$ & $\mathrm{~cm}$ & 1.75 \\
Length & $L_{c h}$ & $\mathrm{~cm}$ & 10.36 \\
Velocity & $V_{c h}$ & $\mathrm{~cm} / \mathrm{s}$ & 1.8 \\
Revolution period of the eddy structures & $T_{c h}$ & $\mathrm{~s}$ & 5.88 \\
Frequency & $f_{c h}$ & $\mathrm{~Hz}$ & 0.17
\end{tabular}

Note. The parameters were calculated by formula (4).

It was revealed in the previous numerical study [19] that the pulsed effect on the flow with a frequency close to $f_{c h}$ leads to a significant increase both in the turbulent kinetic energy of the flow and in the intensity of fluid stirring. This in turn stimulates the heat transfer processes in the bulk.

\section{Conclusion}

The paper discusses the results of an experimental study on the effect of a low-frequency pulsed force of the electromagnetic field on changing the axial flow velocity of a eutectic alloy Galinstan (GaInSn). The axial velocity values were compared with those obtained in applying a constant Lorentz force to the flow. A mathematical model of the flow of Galinstan under a constant force of the EM field was also developed and verified.

The measurements made using a Doppler velocity meter showed that the pattern of the flow forming in the stationary process is consistent with the results 
obtained in other experiments $[8,11,12,14]$, namely, that melt flow mainly consists of two large eddies with opposite directions of rotation relative to each other.

Applying a pulsed Lorentz force in the range of $f_{p}$ values from 0.50 to $10 \mathrm{~Hz}$ has an influence comparable in its intensity with the steady effect. It was discovered that the values of the measured axial fluid velocity are close to those obtained under continuous application of an external impact.

An increase in the value of the fluctuation component of the axial flow velocity is observed under the influence of the pulses at a frequency of $f_{p}=0.05$ $0.20 \mathrm{~Hz}$, and the experimental maximum is reached at $f_{p}=0.10 \mathrm{~Hz}$. It is assumed that the maximum effect can be detected if the frequency of the pulses applied to the Galinstan melt flow is close to $f_{c h}=0.17 \mathrm{~Hz}$.

\section{Acknowledgments}

The study was conducted with the financial support of the German Academic Exchange Service (DAAD) and the Ministry of Education and Science of the Russian Federation.

\section{References}

[1] T. Inaba, T. Kubo, Enhanced heat transfer through oscillatory flow, J. Heat Transf. Jpn. Res. 22 (5) (1993) 480-492.

[2] I.L. Mostinskii, D.I. Lamden, O.G. Stonik, Influence of flow pulsations on the heat and mass transfer with particles, J. High Temp. 21 (4) (1983) 576-582.

[3] E. Baake, B. Nacke, A. Jakovičs, A Umbraško, Heat and mass transfer in turbulent flows with several recirculated flow eddies, Magnetohydrodynamics 37 (1-2) (2001) 13-22.

[4] M. Kirpo, A. Jakovičs, E. Baake, B Nacke, Modeling velocity pulsations in a turbulent recirculated melt flow, Magnetohydrodynamics 42 (2-3) (2006) 207-218.

[5] A. Cramer, C. Zhang, S. Eckert, Local flow structures in liquid metals measured by ultrasonic Doppler velocimetry, Flow Meas. Instrum 15 (3) (2005) 145-153.

[6] G.J. Chen, Y.J. Zhang, Y.S. Yang, Modelling the unsteady melt flow under a pulsed magnetic field, Chin. Phys. B. 22 (12) (2013) 120302-1-120302-5.
[7] H. Branover, E. Golbraikh, A. Kapusta, et al., Novel potentialities of electromagnetic stirring of melts in metallurgy, in: Proceedings of the Joint 15 the Riga and 6 the PAMIR International Conference on Fundamental and Applied MHD, 2005, pp. 31-34.

[8] A. Cramer, V. Galindo, M. Zennaro, Frequency dependence of an alternating magnetic field driven flow, Magnetohydrodynamics 51 (1) (2015) 133-148.

[9] V.I. Dubodelov, V.N. Fixsen, V.K. Pogorsky, et al., Research of the creation process of the modulated oscillations of electromagnetic force and their use processing aluminium alloy, in: Processing of the Third International Simposium on Electromagnetic Processing of Materials, Nagoya, Japan, April 3-6.2000, pp. 67-72.

[10] X. Wang, Y. Fautrelle, J. Etay, R. Moreau, A periodically reversed flow driven by a modulated traveling magnetic field: part I. Experiments with GaInSn, Metall. Mater. Trans. 40B (1) (2009) 82-90.

[11] S. Eckert, P.A. Nikrityuk, D. Rabiger, et al., Efficient melt stirring using pulse sequences of a rotating magnetic field: part I. Flow field in a liquid metal column, Metall. Mater. Trans. B. 38 (6) (2007) 977-988.

[12] Y Takeda, Measurement of velocity profile of mercury flow by ultrasound Doppler shift method, Nucl. Technol. 79 (1) (1987) $120-124$.

[13] Y. Takeda, H Kikura, Flow mapping of the mercury flow, Exp. Fluids 32 (2) (2002) 161-169.

[14] Fluent 6.2 User's Guide (ANSYS. Inc. USA, 2005).

[15] Umbraško A. Heat and mass transfer in electromagnetically driven recirculated turbulent flows (PhD thesis). Riga. University of Latvia, 2011.

[16] E. Baake, A. Mühlbauer, A. Jakovičs, W. Andree, Extension of the $k-\varepsilon$ model for the numerical simulation of the melt flow in induction crucible furnaces, Metall. Mater. Trans. B 26 (3) (1994) 529-535.

[17] M. Albach, J. Patz, H. Roßmanith, D. Exner, A. Stadler, Optimized winding/optimum in power efficiency, Pack Feindaehte 1 (3) (2010) 1-8.

[18] M. Kirpo, A. Jakovičs, E Baake, Characteristics of velocity pulsations in a turbulent recirculated melt flow, Magnetohydrodynamics 41 (2) (2005) 199-210.

[19] D. Musaeva, V. Ilin, E. Baake, V Geza, Numerical simulation of the melt flow in an induction crucible furnace driven by a Lorentz force pulsed at low frequency, Magnetohydrodynamics 51 (4) (2015) 771-785. 